\title{
Elasto-Plastic Load Transfer in Bulk Metallic Glass Composites Containing Ductile Particles
}

\begin{abstract}
DORIAN K. BALCH, ERSAN ÜSTÜNDAG, and DAVID C. DUNAND
In-situ diffraction experiments were performed with high-energy synchrotron X-rays to measure strains in crystalline reinforcing particles ( 5 and 10 vol. pct $\mathrm{W}$ or 5 vol. pct Ta) of bulk metallic glass composites. As the composites were subjected to multiple uniaxial tensile load/unload cycles up to applied stresses of $1650 \mathrm{MPa}$, load transfer from the matrix to the stiffer particles was observed. At low applied loads, where the particles are elastic, agreement with Eshelby elastic predictions for stress partitioning between matrix and particles is found, indicating good bonding between the phases. At high applied loads, departure from the elastic stress partitioning is observed when the particles reach the von Mises yield criterion, as expected when plasticity occurs in the particles. Multiple mechanical excursions in the particle plastic region lead to strain hardening in the particles, as well as evolution in the residual strain state of the unloaded composite.
\end{abstract}

\section{INTRODUCTION}

BULK metallic glasses (BMGs) are multicomponent alloys capable of retaining an amorphous structure upon quenching at low to moderate cooling rates (1 to $100 \mathrm{~K} / \mathrm{s})$, resulting in maximum specimen thickness up to several centimeters. Optimized BMGs have an attractive combination of properties, e.g., very high strength (around $2 \mathrm{GPa}$ ), high

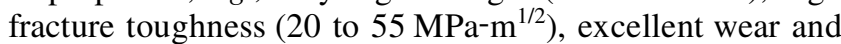
corrosion resistance, and very high elastic limits $(\sim 2 \mathrm{pct}) .^{[1]}$ However, BMGs also have the tendency to fail catastrophically, when unconstrained, by shear banding after very limited plastic deformation at room temperature. Composites, consisting of a BMG matrix and a metallic second phase in the form of particles or fibers, exhibit improved strength and ductility, likely due to load transfer to the stiffer metallic reinforcement and arresting of shear bands by the more ductile metallic reinforcement. ${ }^{[2-5]}$

These BMG composites show important differences in their mechanical behavior as compared to conventional metal matrix composites (MMCs) consisting of a metallic, ductile matrix containing stiffer but brittle reinforcement, e.g., $\mathrm{Al} / \mathrm{SiC}$. The main difference is that the matrix of BMG composites is brittle while that of most MMCs is ductile. This leads to different situations upon cooling from the processing temperature, where usually tensile thermal mismatch strains are developed in the matrix of both types of composites. These strains are easily relaxed by matrix plasticity in MMCs but build up elastically in the BMG matrix, thus decreasing the applied stress at which shear bands are formed upon subsequent tensile loading. Second, upon mechanical loading, the matrix of

DORIAN K. BALCH, formerly with the Department of Materials Science and Engineering, Northwestern University, is Postdoctoral Appointee, Engineered Materials Department, Sandia National Laboratories, Livermore, CA 94551 ERSAN ÜSTÜNDAG, Assistant Professor, is with the Department of Materials Science, California Institute of Technology, Pasadena, CA 91125. Contact e-mail: ersan@caltech.edu DAVID C. DUNAND, Associate Professor, is with the Department of Materials Science and Engineering, Northwestern University, Evanston, IL 60208. Contact e-mail: dunand@ northwestern.edu

Manuscript submitted June 28, 2002. both BMG composites and MMCs transfers some of its load to the stiffer reinforcement. In a BMG composite, however, it is the reinforcing metallic second phase that first exceeds its yield stress during loading, followed by brittle fracture of the matrix. In conventional MMCs, on the other hand, matrix plasticity usually occurs before brittle reinforcement fracture. The load transfer evolution between the matrix and reinforcement upon mechanical loading is thus fundamentally different in the two types of composites.

In traditional MMCs, load partitioning between metallic matrix and ceramic or metallic reinforcement has been investigated during uniaxial loading using in-situ neutron diffraction measurements allowing the independent measurement of volume-averaged, bulk strains in each phase parallel and perpendicular to the loading direction. ${ }^{[6,7,8]}$ The recent availability of high-energy, high-intensity $\mathrm{X}$-rays at third generation synchrotron $\mathrm{X}$-ray facilities has allowed for similar bulk strain measurements in the phases of MMCs..$^{[9,10,11]}$ Advantages of this new technique as compared to neutron diffraction are that small samples can be studied using much shorter data collection times; the associated disadvantage is that the grain size of the phases to be measured must be quite small, typically less than $50 \mu \mathrm{m} \cdot{ }^{[12]}$

The purpose of the present work is to measure by synchrotron X-ray diffraction the average strains in the metallic reinforcement of BMG composites subjected to uniaxial tensile loads both below and above the limit where plastic deformation of the reinforcement occurs. This in-situ deformation data are then used in an Eshelby model to deduce the load transfer between the matrix and reinforcement.

\section{EXPERIMENTAL METHODS}

\section{A. Sample Preparation and Characterization}

Bulk metallic glass with the commercial designation Vitreloy 106 and a nominal composition of $\mathrm{Zr}_{57} \mathrm{Nb}_{5} \mathrm{Al}_{10} \mathrm{Cu}_{15.4} \mathrm{Ni}_{12.6}$, was used as the matrix material. The reinforcement consisted of 
Table I. Physical and Mechanical Properties of BMG, W, and Ta

\begin{tabular}{lcccc}
\hline Phase & $E(\mathrm{GPa})$ & $v$ & $\sigma_{0.2 \text { pct yield }}(\mathrm{MPa})$ & $\mathrm{CTE}^{\left(10^{-6} \mathrm{~K}^{-1}\right)}$ \\
\hline${\text { Vitreloy } 106^{[2]}}$ & 85 & 0.38 & 1800 (compression) & \\
& & & 1200 (tension) & 8.7 \\
Tungsten $^{[14,15]}$ & 410 & 0.28 & 760 & 4.6 \\
Tantalum $^{[14,15]}$ & 185 & 0.34 & 165 & 6.5 \\
\hline
\end{tabular}

tungsten or tantalum particles (supplied by Alfa Aesar, Ward Hill, MA) with nominal diameters of $\approx 15 \mu \mathrm{m}$ and nominal volume fractions of 5 pct (for both tungsten and tantalum composites) and 10 pct (for a second tungsten-containing composite). The three BMG composites were fabricated as described in Reference 13 by induction melting of the BMG matrix and particulate reinforcements, followed by casting into a chilled copper mold. Dog-bone-shaped tensile specimens were then machined with gage sections approximately $10-\mathrm{mm}$ long, 1-mm wide, and 1-mm thick. Values for various physical properties of both matrix and reinforcing phases are summarized in Table I. ${ }^{[2,14,15]}$

Scanning electron microscopy (SEM) was performed to characterize particle size, volume fraction, and distribution on polished sections of the composites. Particle sizes were measured using the linear intercept method. Volume fractions were calculated from particle area measurements using digital imaging techniques. Microchemical analysis using wavelength-dispersive spectrometry (with elemental standards) on an electron microprobe was also performed to quantify interdiffusion between matrix and reinforcement. Both microscopy and chemical analysis were carried out on a JEOL $^{*} 733$ SEM.

*JEOL is a trademark of Japan Electron Optics Ltd., Tokyo.

\section{B. Diffraction and Mechanical Experiments}

Diffraction measurements were made at the DuPontNorthwestern-Dow Collaborative Access Team (DND-CAT) at Sector 5 of the Advanced Photon Source (Argonne National Laboratory, Argonne, IL). In-situ uniaxial tensile tests were performed using a small screw-driven tensile loading system, described in Reference 16. The tensile stress on the composites was varied between 0 and $1650 \mathrm{MPa}$ in steps of 75 to $300 \mathrm{MPa}$, and was held constant during the $\mathrm{X}$-ray exposures. The 5 and 10 pct $\mathrm{W}$ composites were loaded to approximately $1000 \mathrm{MPa}$, unloaded to $0 \mathrm{MPa}$, and immediately reloaded to fracture at $1650 \mathrm{MPa}$ for both samples. The 5 pct Ta composite was initially loaded to $1000 \mathrm{MPa}$ and unloaded; at a later date, nearly 7 months later, the sample was reloaded to $1200 \mathrm{MPa}$, unloaded, and loaded a third time to fracture at $1585 \mathrm{MPa}$.

The general set up for these experiments has been described in detail in Reference 9, and is shown schematically in Figure 1. Each sample, subjected to a constant uniaxial load, was irradiated for 600 seconds with a monochromatic $65 \mathrm{keV}(\lambda=0.019 \mathrm{~nm}) \mathrm{X}$-ray beam with a square crosssectional area of $0.4 \times 0.4 \mathrm{~mm}$. The Debye-Scherrer $\{110\}$ diffraction cone from the reinforcing particles present in the diffraction volume of $\approx 0.16 \mathrm{~mm}^{3}$ was recorded using a charge coupled device (CCD) camera. Also recorded was the $\{110\}$ Debye-Scherrer cone from an iron powder

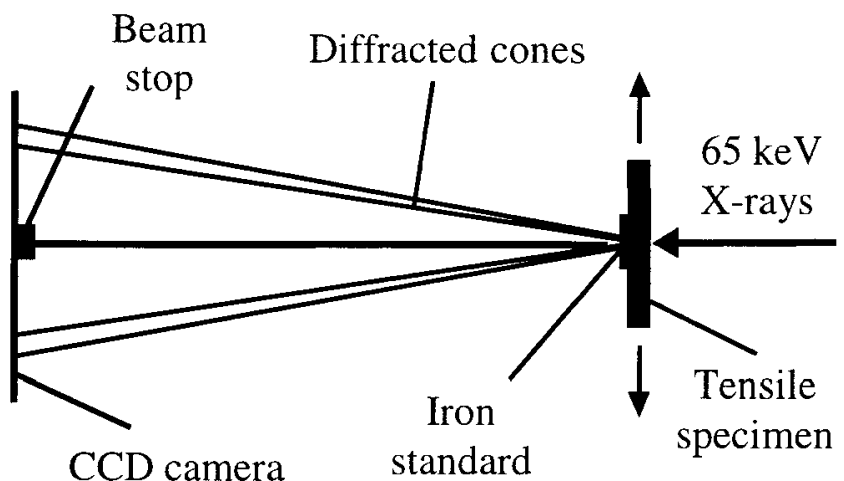

Fig. 1-X-ray measurement setup.

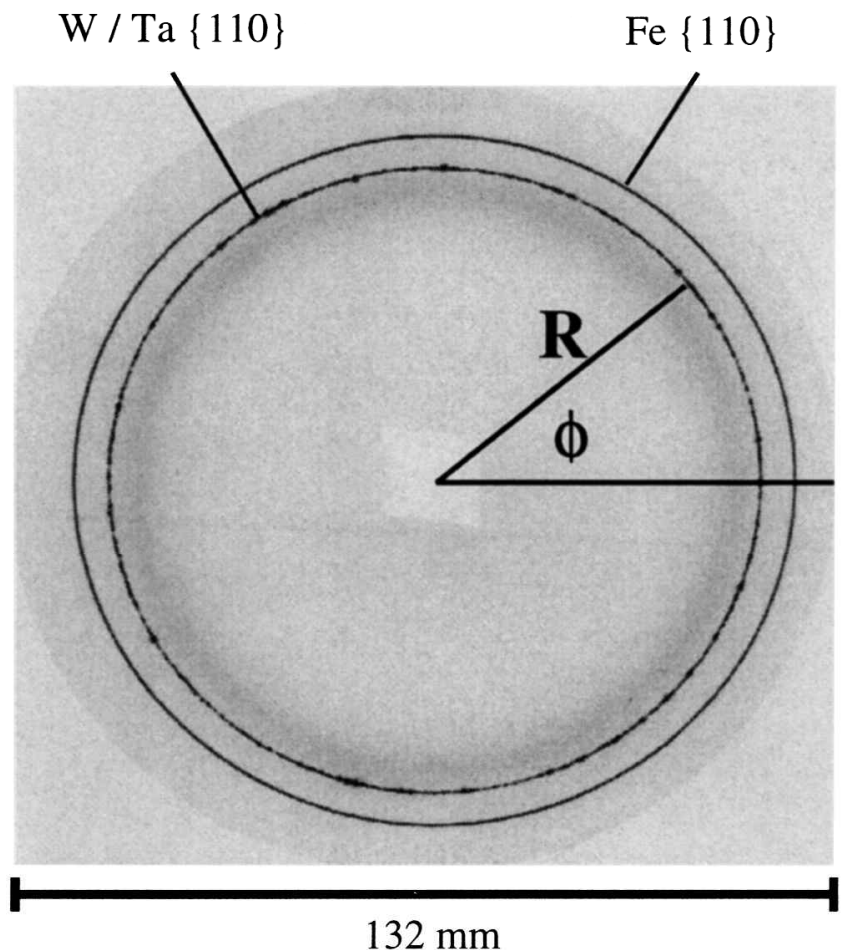

Fig. 2-Typical diffraction pattern, showing the reinforcement and standard rings; $R$ and $\phi$ are defined in Section III.

standard attached to the sample for calibration purposes. The two resulting concentric rings are shown in Figure 2 for the 10 pct $W$ composite. The CCD camera (from MAR Inc., Evanston, IL) was situated $618 \mathrm{~mm}$ away from the sample and had a 132-mm-diameter detector with 16 bit intensity readings over an orthogonal array of 64.4 by $64.4 \mu \mathrm{m}$ pixels. 


\section{ANALYSIS}

As described in Reference 9, the lattice plane spacing $d^{\circ}$ of a stress-free sample is related at small Bragg angles to the X-ray wavelength $\lambda$, camera distance $L$, and diffracted ring diameter $D^{\circ}$ :

$$
d^{\circ} \approx \frac{2 \lambda L}{D^{\circ}}
$$

Application of a uniaxial tensile stress increases the lattice spacing of atomic planes oriented perpendicular to the loading axis and, through the Poisson effect, decreases the lattice spacing of planes parallel to the loading axis. This results in the distortion of the diffraction rings from (ideally) perfect circles into ellipses with shorter diameter $D^{\text {axial }}$ in the loading direction and longer diameter $D^{\text {transverse }}$ in the perpendicular direction. Strains $\varepsilon=\left(d-d^{\circ}\right) / d^{\circ}$ in the axial and transverse directions can then be calculated using the following expression:

$$
\varepsilon \approx \frac{D^{\circ}-D}{D} \approx \frac{D^{\circ}-D}{D^{\circ}}
$$

As discussed in Reference 9, the use of a stress-free powder reference standard attached to the sample eliminates strain errors caused by fluctuations in X-ray wavelength or camera distance. Combined analysis of the specimen and reference powder rings provides strain values using the following equation:

$$
\varepsilon_{s} \approx 1-\frac{D_{s}}{D_{s}^{\circ}} \cdot \frac{D_{c}^{\circ}}{D_{c}}
$$

where subscripts $S$ and $C$ refer to sample and calibration powder.

Determination of lattice strains from the diffracted rings recorded by the CCD camera consists of the following steps.

1. Identification of the center of the ring, using commercial 16-bit image viewing software.

2. Re-binning of the orthogonally-binned intensity data into radius/angle $(R / \phi)$ polar coordinates with respect to the ring center (as shown in Figure 2), done on 10,000 to 12,000 radial lines in $\phi$ steps of 0.030 to $0.036 \mathrm{deg}$ using a custom program.

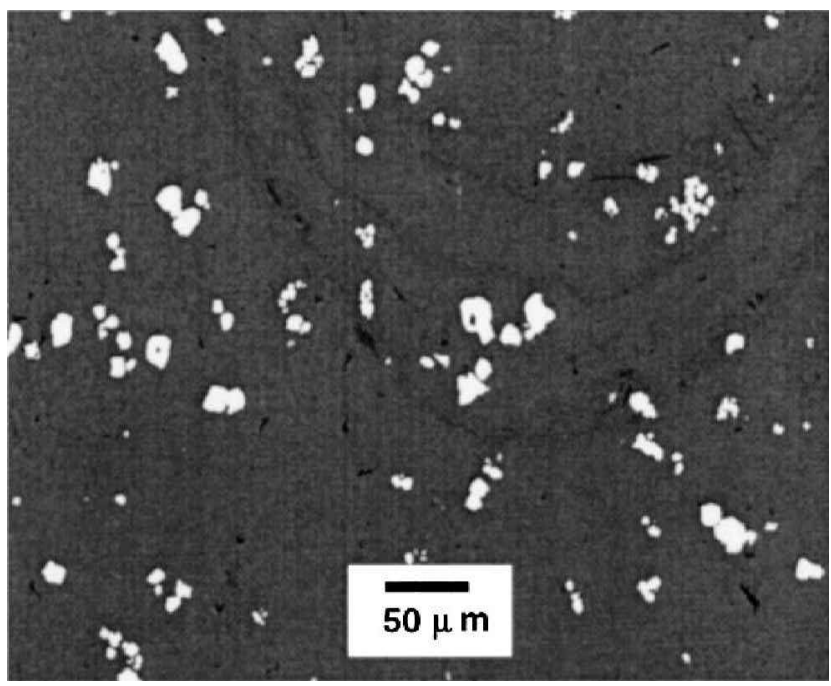

(a) 3a. Gaussian peak fitting in $R$ at each angular position around the ring, and

$3 \mathrm{~b}$. addition of Gaussian peak center positions of opposite angular positions to obtain the ring diameter $D$ as a function of $\phi$ from 0 to $180^{\circ}$, both done using a custom program.

4. Determination of the ring diameters by fitting an ellipse to the $D(\phi)$ data, using the approximation for ellipses with nearly equal semiaxes $a$ and $b$ (i.e., $D(\phi)=a+(b-a)$ $\left.\sin ^{2} \phi\right)$. Performing a linear least-squares fit through a $D(\phi) v s \sin ^{2} \phi$ plot provides $D^{\text {axial }}$ at $\sin ^{2} \phi=1$ (parallel to the loading axis) and $D^{\text {transverse }}$ at $\sin ^{2} \phi=0$ (perpendicular to the loading axis).

5. Calculation of lattice strains $\varepsilon^{\text {axial }}$ parallel to the loading axis and $\varepsilon^{\text {transverse }}$ normal to the loading axis using Eq. [3].

The primary source of error in such an analysis is the least-squares fitting of the $D(\phi) v s \sin ^{2} \phi$ data; this produces uncertainties in $D^{\text {axial }}$ and $D^{\text {transverse }}$ that are a function of the smoothness and intensity of the diffraction rings. Other sources of error are slight misalignment of the sample, relative to both the loading axis and the CCD camera; rotation of the sample around the loading axis during testing in the single-screw-driven tensile loading system; and, for axial strains $\left(\sin ^{2} \phi=1\right)$ only, an offset equal to the Bragg angle (approximately $2.5 \mathrm{deg}$ ) between the loading axis and the measured lattice planes. This offset exists for transverse strains as well, but is not a source of error due to the isotropy of the strains perpendicular to the loading axis. This combination of uncertainties, assuming generous levels of experimental error ( 2 deg for each type of sample misalignment and 5 deg of potential sample rotation), equates to uncertainties in the reported axial and transverse strains of \pm 75 and $\pm 65 \mu \varepsilon$ (microstrain units equal to a strain of $10^{-6}$ ), respectively.

\section{RESULTS}

Representative SEM micrographs of the composites are shown in Figures 3(a) and (b). The average particle sizes were approximately 12 to $14 \mu \mathrm{m}$ for the $\mathrm{W}$ and $9 \mu \mathrm{m}$ for

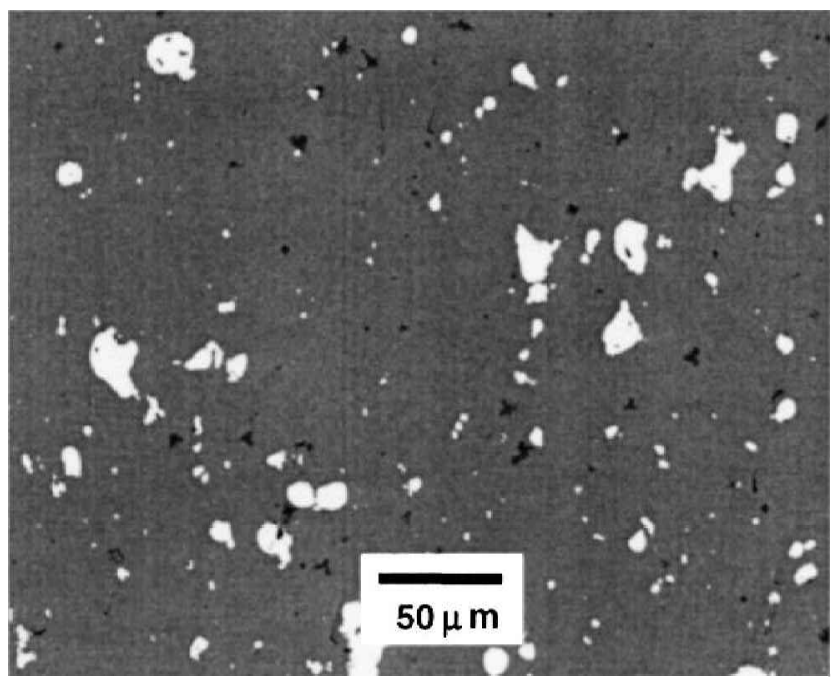

(b)

Fig. 3-Backscattered electron SEM micrographs: (a) 5 pct W composite and (b) 5 pct Ta composite. The bright regions are the metallic particles embedded in a BMG matrix (gray). The dark regions are surface pits due to particle pullout during polishing. 
Table II. Chemical Analysis by Electron Microprobe (Atomic Percent with \pm 0.2 Atomic Percent Error)

\begin{tabular}{|c|c|c|c|c|c|c|c|c|}
\hline Sample & Region & $\mathrm{Zr}$ & $\mathrm{Nb}$ & $\mathrm{Al}$ & $\mathrm{Cu}$ & $\mathrm{Ni}$ & W & $\mathrm{Ta}$ \\
\hline 5 pct $\mathrm{Ta}$ & $\begin{array}{l}\text { matrix } \\
\text { particle }\end{array}$ & $\begin{array}{r}56.9 \\
0.4\end{array}$ & $\begin{array}{l}3.4 \\
0.1\end{array}$ & $\begin{array}{r}10.3 \\
0.1\end{array}$ & $\begin{array}{r}15.4 \\
0.4\end{array}$ & $\begin{array}{r}12.7 \\
0.4\end{array}$ & $\begin{array}{l}0.0 \\
0.0\end{array}$ & $\begin{array}{r}1.3 \\
98.7\end{array}$ \\
\hline
\end{tabular}

the Ta particles, and the particle volume fractions were 5.0 pct and 12.7 pct for the $\mathrm{W}$ composites and 5.2 pct for the Ta composite, as measured by image analysis. Some agglomeration of particles was observed, as seen in Figure 3 . In the following analysis, the nominal volume fractions of 5.0 pct (for $\mathrm{W}$ and Ta) and 10.0 pct (for W) are used. The results of electron microprobe analysis are summarized in Table II, and indicate minimal interdiffusion between matrix and particles in the $\mathrm{W}$ composites and some interdiffusion in the Ta composite.

The strain evolution in the axial and transverse directions for the tungsten and tantalum particles is depicted in Figures 4 through 7 . In all figures of strain data, axial strains are denoted by filled symbols, transverse strains are denoted by hollow symbols, and the starting point is marked with a capital S. Figure 4 shows the axial and transverse strains for the 5 pct $\mathrm{W}$ composite, and Figure 5 provides the same information for the 10 pct W composite. Figure 6 shows the axial and transverse strains during the first loading cycle of the 5 pct Ta composite; the second and third loading cycles are shown in Figures 7(a) and (b). In all cases, the applied stress on the composite is plotted against the lattice strain in the particles, incorporating residual thermal strains, as discussed in the following section.

Slight hysteresis was seen during unloading and reloading of the W-containing composites; this was on the order of 200 to $300 \mu \varepsilon$ for the 5 pct W composite and closer to $600 \mu \varepsilon$ for the 10 pct $\mathrm{W}$ composite. Based on the linearity of the reloading data, it would appear that the unloading data are the source of the hysteresis. A possible explanation is uneven unloading in the single-screw driven tensile rig: some backlash or sample torsion may have occurred, slightly altering the stress state, and therefore elastic particle strains, in the composites. The large hysteresis during unloading and subsequent reloading of the 5 pct Ta composite is due to particle yielding, as discussed later.

\section{DISCUSSION}

\section{A. Microstructure}

Reaction or interdiffusion between the BMG matrix and the metallic particles could have occurred during processing at elevated temperatures, affecting load transfer through interface fracture (in the case of reaction products) or solidsolution hardening (in the case of interdiffusion). Micrographs (Figure 3) display a matrix/particle interface free of reaction products, while microprobe results (Table II) show negligible diffusion of matrix elements into the particles of the

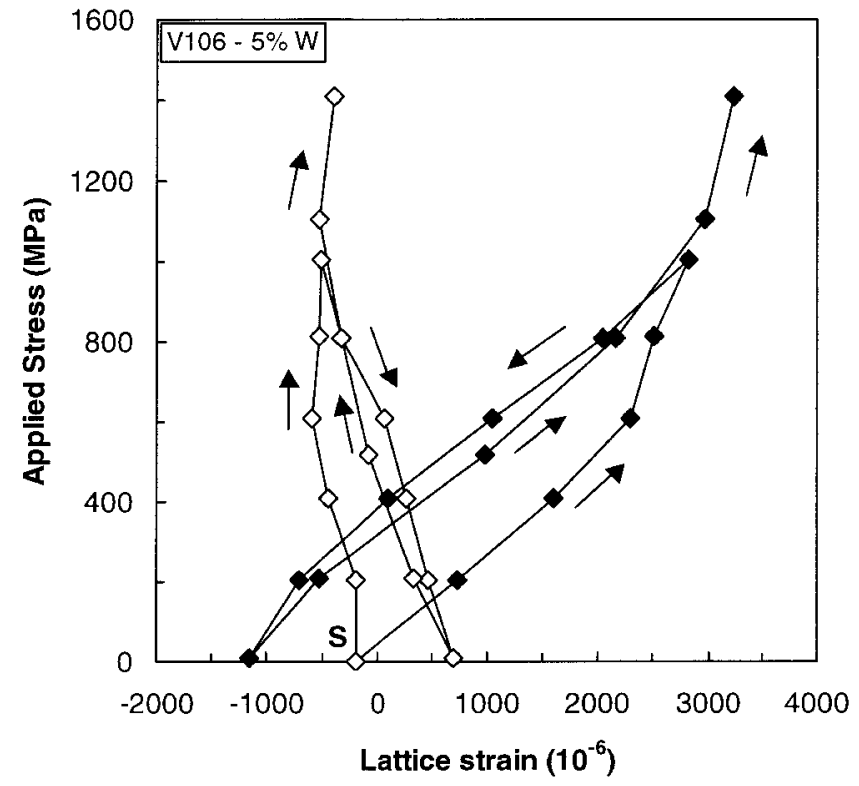

Fig. 4-Applied stress vs elastic lattice strain for the 5 pct W composite. Filled symbols denote axial strains, hollow symbols denote transverse strains, and the starting point is marked with an "S."

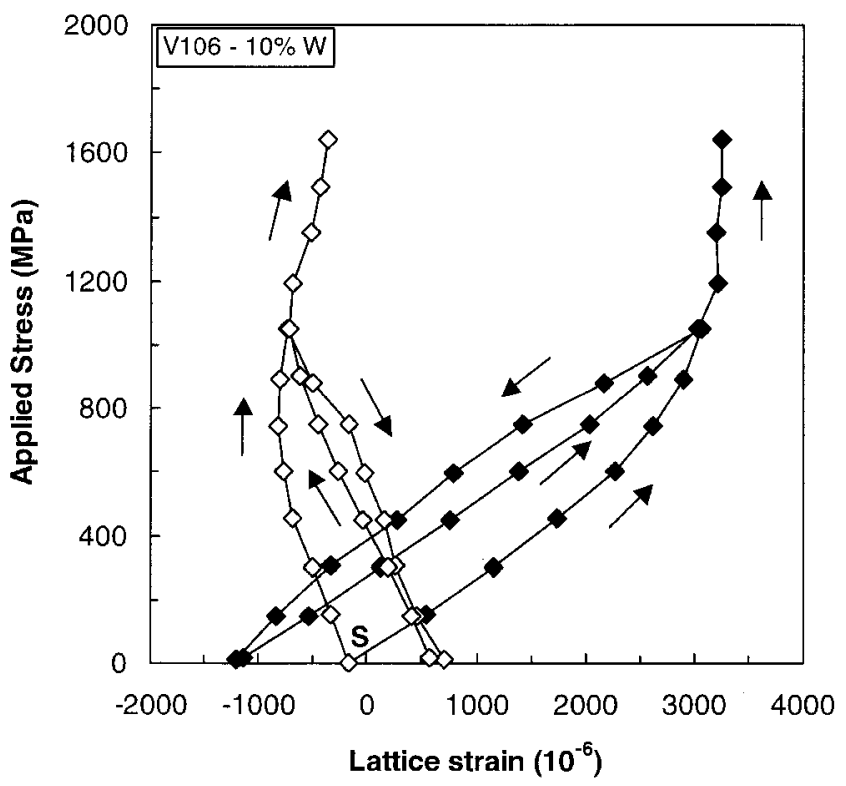

Fig. 5-Applied stress $v s$ elastic lattice strain for the 10 pct W composite. Filled symbols denote axial strains, hollow symbols denote transverse strains, and the starting point is marked with an "S." 
W-containing composites. Slightly more diffusion occurred in the Ta composite, both of matrix elements into the particles and of $\mathrm{Ta}$ into the matrix. It is assumed that in both cases the amount of interdiffusion present does not significantly influence particle yield stresses and strain-hardening behavior by solid-solution strengthening. One effect of matrix element diffusion into the $\mathrm{W}$ and Ta particles is a probable shift in the lattice spacings of the $\mathrm{W}$ and Ta grains. As this chemical effect cannot be separated from the thermal residual strains, the residual strains present prior to testing were calculated, rather than measured.

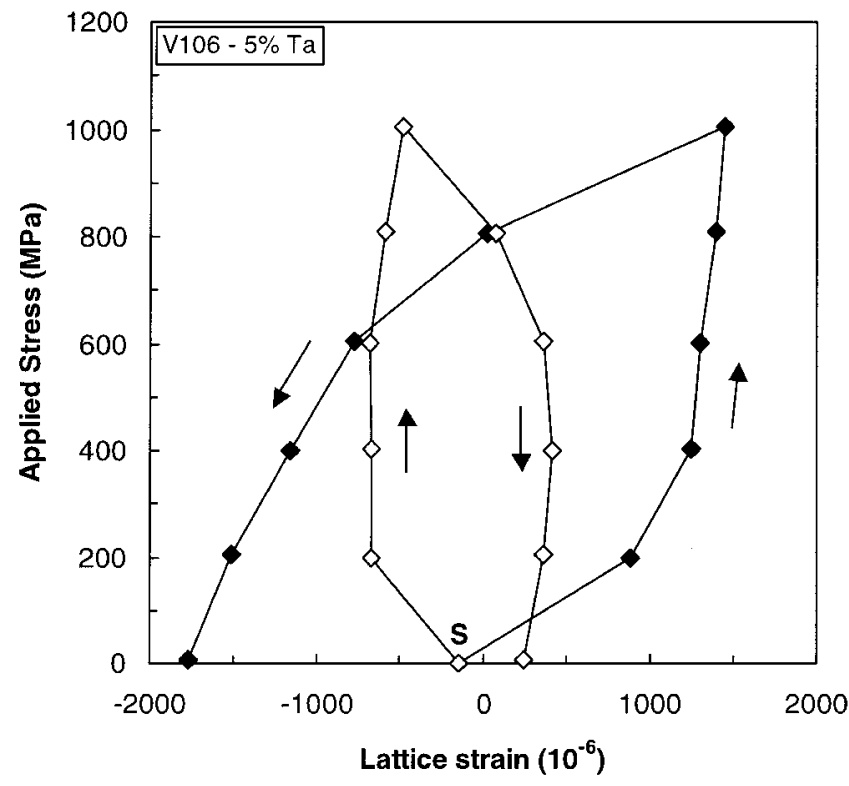

Fig. 6-Applied stress $v s$ elastic lattice strain for the first mechanical cycle of the 5 pct Ta composite. Filled symbols denote axial strains, hollow symbols denote transverse strains, and the starting point is marked with an "S."

\section{B. Thermal Residual Strains}

During cooling from fabrication, residual stresses in matrix and particles are expected to accumulate due to thermal expansion mismatch between matrix and reinforcement. The average thermal expansion coefficients of the BMG matrix, Ta, and $\mathrm{W}$ are 8.7, 6.5, and $4.6 \times 10^{-6} \mathrm{~K}^{-1}$, respectively ${ }^{[2,15]}$ between room temperature and $420^{\circ} \mathrm{C}$, the glass transition temperature of the matrix ${ }^{[2]}$ The higher value of the matrix CTE leads to compressive stresses in the particles balanced by, on average, tensile stresses in the matrix. Assuming the glass transition temperature of the matrix as the stress-free temperature, ${ }^{[17]}$ the residual strains present in the particles at room temperature are calculated with the Eshelby equivalent inclusion method ${ }^{[18]}$ to be -190 , -180 , and $-140 \mu \varepsilon$ for the 5 pct W, 10 pct $\mathrm{W}$, and 5 pct Ta composites, respectively. These strains are strictly hydrostatic in nature because the particles were assumed to be spherical in shape, and can thus be added to the lattice axial and transverse strains measured by diffraction. All strain results shown in Figures 4 through 6 incorporate this correction, which leads to a small systematic shift of the data points along the strain axis, without altering the shapes of the curves.

Using the elastic properties of $\mathrm{W}$ and $\mathrm{Ta},{ }^{[14]}$ the thermal residual strains correspond to hydrostatic particle stresses at room temperature of $-170,-160$, and $-85 \mathrm{MPa}$ for the 5 pct W, 10 pct W, and 5 pct Ta composites, respectively. The corresponding average tensile stresses in the BMG matrix are $8.8,17$, and $4.5 \mathrm{MPa}$, negligibly small as compared to the matrix yield stress of $1200 \mathrm{MPa} \cdot{ }^{[2]}$

\section{Strains due to External Loads}

\section{BMG-5 pct, 10 pct W Composites}

The lattice strain evolutions of the 5 and 10 pct W composites are qualitatively similar. As shown in Figures 4 and

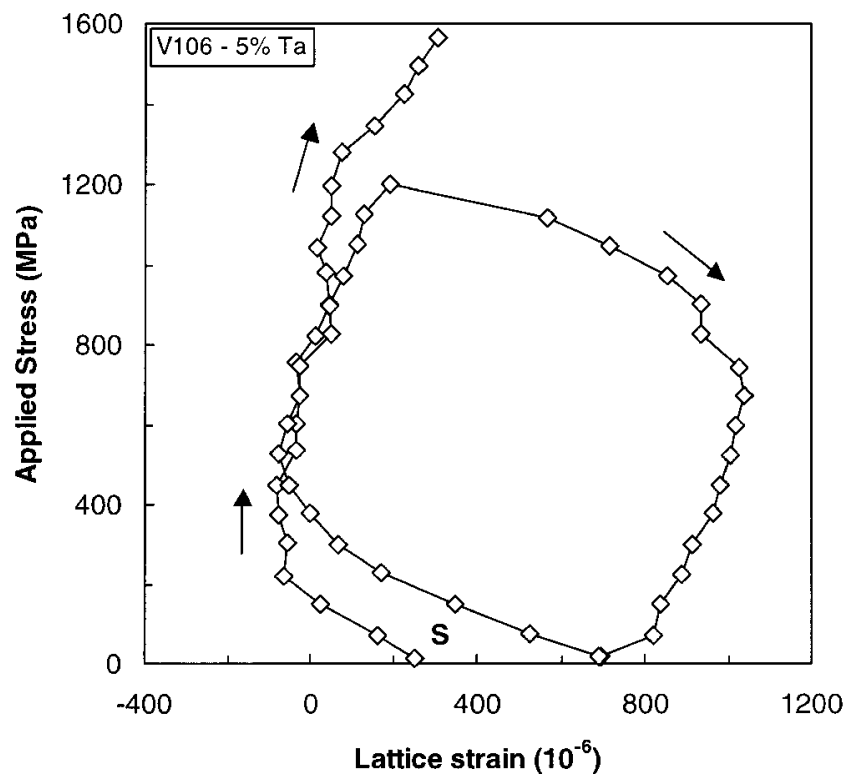

(b)

Fig. 7-Applied stress $v s$ elastic lattice strain for the second and third mechanical cycles of the 5 pct Ta composite: $(a)$ axial and (b) transverse. Filled symbols denote axial strains, hollow symbols denote transverse strains, and the starting point is marked with an "S." 
5 in the axial direction, the initial response is linear up to an applied stress slightly above $600 \mathrm{MPa}$. Beyond this point, the applied stress-lattice strain curves turn upward, indicating that comparatively less stress is carried by the particles as the applied stress is increased. The opposite behavior has been observed in many conventional MMCs and has been conclusively attributed to the onset of plasticity in the metallic matrix, which increases the mismatch with the elastic reinforcement. ${ }^{[18]}$ We interpret the deflection in Figures 4 and 5 in a similar manner, except that plasticity is occurring in the particles within an elastic matrix. Since plasticity is occurring in the elastically stiffer phase, the degree of mismatch decreases, leading to decreased load transfer to the particles. The qualitative effect, however, is similar, i.e., the degree of transferred load changes due to a change in mismatch between phases.

At the maximum applied stresses of $\sim 1000 \mathrm{MPa}$, the particles in both composites are thus well beyond their yield point, while the matrix is still behaving in an elastic fashion. During subsequent unloading from 1000 to $0 \mathrm{MPa}$, on average, both matrix and particles behave elastically, with the $\mathrm{W}$ axial strains becoming compressive below an applied stress of roughly $350 \mathrm{MPa}$. The unloading curves in Figures 4 and 5 remain linear to $0 \mathrm{MPa}$, indicating that the yield stress in compression was not reached. The residual axial strains present after unloading are -1150 and $-1210 \pm 75$ $\mu \varepsilon$ for the 5 pct and 10 pct $\mathrm{W}$ composites. Although the W particles bear a slightly lower fraction of the applied stress in the 10 pct composite, the slightly higher (1050 vs $1000 \mathrm{MPa}$ ) stress reached by the 10 pct W composite during initial loading gives rise to the larger residual strain upon unloading.

Upon subsequent reloading of the composites, Figures 4 and 5 show a linear behavior in the axial direction up to $1000 \mathrm{MPa}$ applied stress. This indicates that the $\mathrm{W}$ particles are elastic up to this stress, as expected from strain hardening due to the previous loading. Beyond the applied stress of $1000 \mathrm{MPa}$, the curves exhibit an abrupt change of slope and follow the trends observed during the first loading. This can again be interpreted by plastic deformation of the particles, which continues up to composite failure. For both composites, the slopes of the stress-strain curves in the three elastic regions (loading, unloading, and reloading) are the same within experimental error. This is evidence that no significant interfacial or particle fracture occurred, which would have led to a reduction in load transfer efficiency observable by a change in elastic slope in Figures 4 and 5 .

Figures 4 and 5 also show the $\mathrm{W}$ lattice response in the transverse direction. During initial loading, the transverse strains increase linearly with applied stresses as expected if the reinforcement is elastic, but strains are negative due to Poisson contraction. As expected from the axial strain results, a deflection is observed at the same stress level (approximately $600 \mathrm{MPa}$ ) where plasticity was identified to begin. In agreement with that interpretation, the transverse stresses carried by the reinforcement in the plastic regime are lower than in the elastic regime. For both composites, the slope of the stress-strain trajectory changes sign, i.e., the particle transverse strains become increasingly tensile at high applied stress, despite the expected Poisson contraction. This unexpected effect can be assigned to the change in effective bulk (rather than elastic lattice) Poisson's ratio of tungsten from $v=0.28$ to 0.5 due to conservation of volume upon plasticity. Thus, the Poisson's ratio mismatch between the elastic matrix $(v=0.38)$ and the plastic particles $(v=0.5)$ leads to tensile stresses in the transverse directions during continued loading to $\sim 1000 \mathrm{MPa}$.

During subsequent unloading to $0 \mathrm{MPa}$ and reloading to $1000 \mathrm{MPa}$, the particles behave elastically in the transverse directions within experimental error, in agreement with the behavior in the axial direction. Particle residual transverse strains after unloading from $\sim 1000 \mathrm{MPa}$ were 700 and $710 \pm 65 \mu \varepsilon$ for the 5 and 10 pct W composites, respectively. Continued loading beyond $1000 \mathrm{MPa}$ results in continuation of the trends observed in the plastic region, with the particles in both composites experiencing triaxial tensile loading.

\section{BMG-5 pct Ta Composite}

The axial and transverse strains during the first loading and unloading of the composite are shown in Figure 6. The axial and transverse strains both follow qualitatively the same paths described for the tungsten-reinforced composites, except for two differences. First, on loading, the onset of plasticity in the Ta particles occurs at a much lower applied stress near $200 \mathrm{MPa}$. Second, upon unloading, after an initial region of elastic behavior is observed down to approximately $600 \mathrm{MPa}$ applied stress, a second deflection is observed, which can be interpreted as onset of compressive plastic deformation. Both these observations can be qualitatively explained by the fact that the uniaxial yield stress of tantalum (approximately $165 \mathrm{MPa}$ ) is considerably lower than that of tungsten (approximately $760 \mathrm{MPa}) .{ }^{[15]}$ Thus, the tantalum particles yield, first in tension during loading above an applied stress of approximately $200 \mathrm{MPa}$ and then in compression upon unloading below an applied stress of approximately $600 \mathrm{MPa}$. As with the W composites, the transverse strains upon loading undergo the same reversal in direction due to the change in the macroscopic Poisson's ratio of tantalum from $v=0.34$ to 0.5 . Upon unloading, an opposite and symmetrical reversal is observed in the transverse strain trajectory at an applied stress of $600 \mathrm{MPa}$, confirming the previous conclusion based on axial strains that plasticity occurs in compression during unloading. At the end of the first load-unload cycle, the residual strains are $-1770 \pm 75 \mu \varepsilon$ and $250 \pm 65 \mu \varepsilon$ in the axial and transverse directions, respectively.

The axial and transverse strain response of the Ta-BMG sample during the second and third loading cycles ( $\begin{array}{ll}0 & 1200\end{array}$

$01585 \mathrm{MPa}$ ), shown in Figures 7(a) and (b), exhibits the same behavior seen during the first cycle, i.e., initial elastic behavior followed by plastic deformation during both loading and unloading. The slopes of the stress-strain curves in the elastic regions are again constant within experimental error, indicating that large-scale particle fracture or debonding is not occurring. In Figures 7(a) and (b), the first point of the second loading cycle was placed at the same position as the last point of the first cycle (Figure 6); however, the second and third loading cycles occurred nearly 7 months after the first cycle, and as a result the absolute position of the applied stress vs lattice strain curves along the strain axis may have shifted due to relaxation.

\section{Load Transfer}

As both tungsten and tantalum have significantly higher stiffnesses than the BMG matrix (by a factor of 4.8 and 2.2, 
respectively, (Table I)) considerable load transfer is expected during composite deformation: the particles carry stresses higher than the applied stress on the composite, thereby lowering the mean stress carried by the matrix. This mechanism can be modeled by the Eshelby equivalent inclusion method. ${ }^{[18]}$ Figures 8(a) through (c) show elastic Eshelby predictions and experimental values of applied stress $v s$ axial and transverse microstrain for all loading cycles of the three composites. The experimental data have been shifted along the strain axis to originate at the same residual strain. The Eshelby predictions, based solely on the elastic constants and volume fractions of each phase, are in excellent agreement with the measured elastic strains for the $\mathrm{W}$ composites in their elastic range. The 5 and 10 pct $\mathrm{W}$ lattice strain

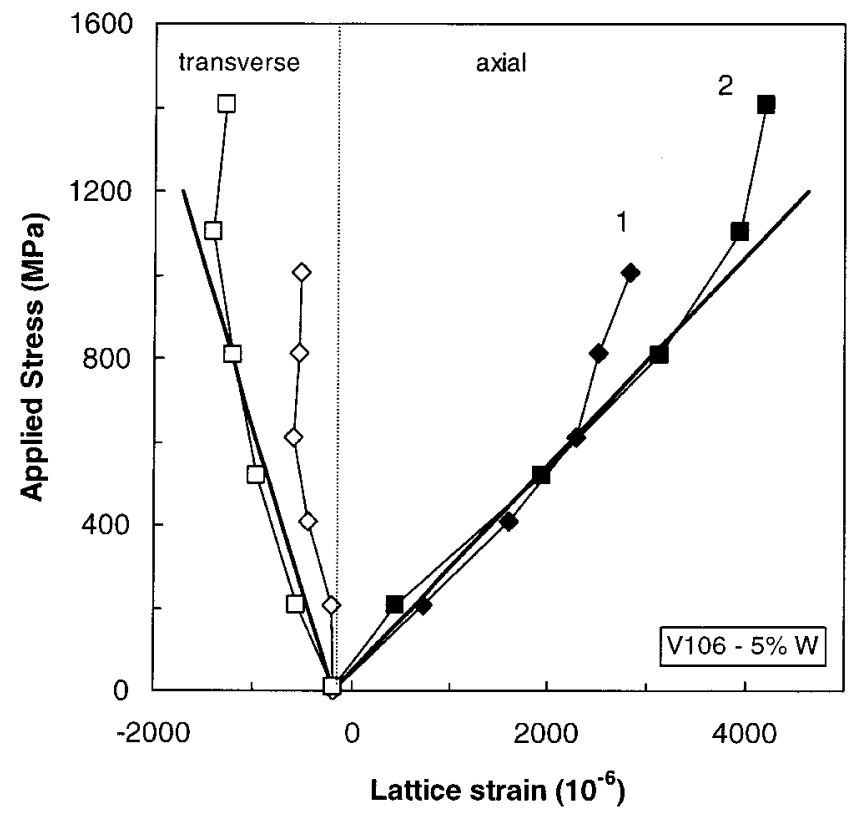

(a) curves from the first and second loadings overlap, with the second loading curves matching the Eshelby predictions to higher values of applied stress, as expected from strain hardening. The agreement between experiment and theory in the 5 pct Ta composite is affected by the fact that the Ta particles most likely underwent yielding slightly below $200 \mathrm{MPa}$ applied stress.

The magnitude of matrix unloading due to load transfer at $600 \mathrm{MPa}$ applied stress (around the initiation of particle plastic flow) can be calculated as the difference between the applied stress and the average matrix axial stress calculated using the measured axial and transverse strains, as explained in the following section. Without including the matrix residual tensile stress, the matrix unloading is 17 and $33 \mathrm{MPa}$ in the

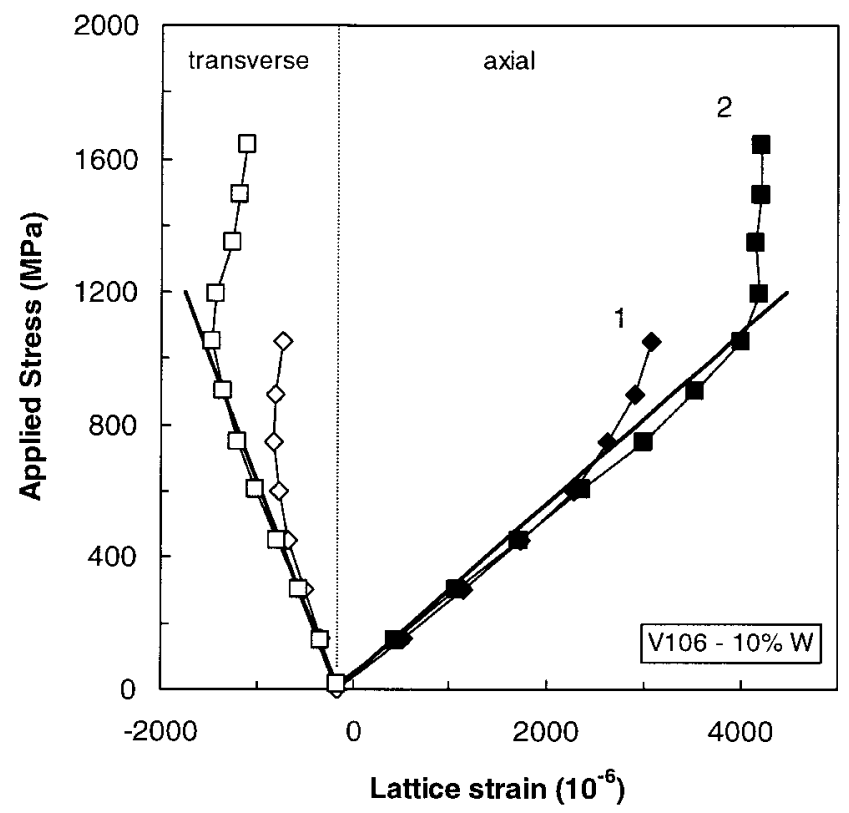

(b)

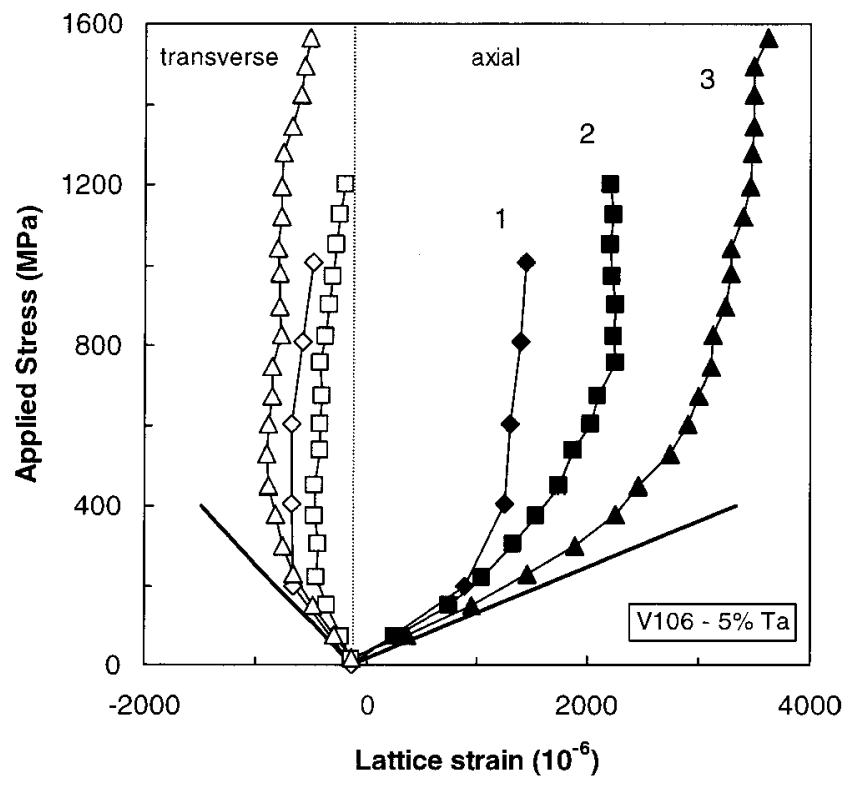

(c)

Fig. 8-Applied stress $v s$ elastic lattice strain (transverse and axial) as measured during loading and as predicted by the elastic Eshelby calculations (loading sequence is indicated with numerals, and Eshelby predictions are shown with lines): (a) $5 \mathrm{pct} \mathrm{W},(b) 10 \mathrm{pct} \mathrm{W}$, and (c) 5 pct Ta. 
5 and 10 pct $\mathrm{W}$ composites, respectively; these values are reduced to near zero by the thermal residual stresses. After the initiation of plastic flow in the particles, however, load transfer efficiency is considerably reduced. For example, at an applied stress of $1644 \mathrm{MPa}$ on the 10 pct $\mathrm{W}$ composite, just prior to sample failure, the axial stress in the W particles is $1462 \mathrm{MPa}$, and by stress balance, the average axial stress in the matrix is $1665 \mathrm{MPa}$. Thus, at very high applied stresses, the plastically deformed particles are in fact increasing the load on the matrix, rather than decreasing it as seen in traditional MMCs with stiff, elastic reinforcement. Then, the sole benefit provided by the particles is the arresting of propagating shear bands, considered to be the reason these composites reach tensile stresses well in excess of the tensile failure stress $(1200 \mathrm{MPa})$ of the unreinforced matrix. ${ }^{[2,19]}$

\section{Particle Yielding}

For uniaxial deformation, the principal strains $\varepsilon_{1}$ (along the direction of applied stress) and $\varepsilon_{2}=\varepsilon_{3}$ (perpendicular to that direction) correspond to the measured axial and transverse strains, respectively. The axial and transverse principal stresses $\sigma_{1}, \sigma_{2}$, and $\sigma_{3}$ in the particles can then be calculated from these principal strains according to ${ }^{[20]}$

$$
\begin{aligned}
& \sigma_{1}=\frac{E}{1+v} \varepsilon_{1}+\frac{v E}{(1+v)(1-2 v)}\left(\varepsilon_{1}+\varepsilon_{2}+\varepsilon_{3}\right)[4 \mathrm{a}] \\
& \sigma_{2}=\sigma_{3}=\frac{E}{1+v} \varepsilon_{2}+\frac{v E}{(1+v)(1-2 v)}\left(\varepsilon_{1}+\varepsilon_{2}+\varepsilon_{3}\right)[4 \mathrm{~b}]
\end{aligned}
$$

where $E$ is the Young's modulus and $v$ the Poisson's ratio. These stresses can in turn be used to determine the particle von Mises effective stress, $\sigma_{\text {eff }}{ }^{[20]}$

$$
\left.\sigma_{\text {eff }}=\frac{1}{\frac{1}{2}}\left[\sigma_{1}-\sigma_{2}\right)^{2}+\left(\sigma_{2}-\sigma_{3}\right)^{2}+\left(\sigma_{3}-\sigma_{1}\right)^{2}\right]_{1 / 2}
$$

The residual stresses caused by thermal expansion mismatch are assumed to be hydrostatic due to the near spherical shape of the particles, and therefore have no effect on the von Mises effective stress. In using Eq. [4], it is assumed that $\mathrm{W}$ and Ta can be treated as isotropic materials, which is reasonable given their low elastic anisotropy factors (1.01 and 1.56, respectively) ${ }^{[14]}$ Figures 9(a) through (c) show the values of $\sigma_{\text {eff }}$ calculated at each applied stress for the $\mathrm{W}$ composites and during the first mechanical cycle (loading and unloading) of the Ta composite. Reinforcement yielding is expected when the von Mises effective stress reaches the uniaxial yield stress, for which literature values $^{[15]}$ are also shown in Figure 9. It is apparent from Figures 9(a) and (b) that during loading of the 5 and 10 pct W composites, the values of $\sigma_{\text {eff }}$ increase linearly with applied stress and depart from the linear behavior at a value in the range of 800 to $1000 \mathrm{MPa}$, slightly above the uniaxial tungsten yield stress of $760 \mathrm{MPa}$. This observation confirms quantitatively that yielding is occurring in the particles at an applied stress of approximately $600 \mathrm{MPa}$. Following yield, the slopes of the $\sigma_{\text {eff }}$ curves decrease continuously, as elastic load transfer to the plastic particles becomes less effective. During unloading, $\sigma_{\text {eff }}$ decreases linearly to $0 \mathrm{MPa}$ and then increases again with a slope of the same magnitude but opposite sign, until the applied stress is zero. At this point, the uniaxial yield stress is not reached so that the $\mathrm{W}$ particles are in net compression but remain in the elastic region. During the second loading, the effective stresses retrace the unloading trajectory, as expected, thus remaining linear up to approximately 1100 to $1200 \mathrm{MPa}$, a yield stress easily reached in strain-hardened tungsten. ${ }^{[15]}$ Further loading into the plastic regime leads to a near zero increase in effective stress. Although no macroscopic strain data could be obtained during these experiments, tensile macroscopic stress-strain curves of Vitreloy 106 containing 5 pct $\mathrm{W}$ particles previously published by Reference 2 show a deflection from linearity at stresses of 600 to $700 \mathrm{MPa}$, in good agreement with our observation of particle yielding at an applied stress of $600 \mathrm{MPa}$ in our 5 pct W composites.

While the tungsten particle residual stresses are hydrostatic prior to deformation and the effective stress is thus zero, the effective particle residual stress after the first mechanical cycle reaches values of about $-600 \mathrm{MPa}$ due to plastic mismatch (Figures 9(a) and (b)), corresponding to a small effective tensile residual stress of 30 and $60 \mathrm{MPa}$ in the BMG matrix of the 5 and 10 pct $\mathrm{W}$ composites, respectively. Matrix stress values near the particle interface are expected to be considerably higher, as the calculated values are volume averages. These residual stresses due to mechanical cycling increase with increasing volume fractions of metallic reinforcement, thus significantly affecting the applied stress at which fracture of the BMG matrix will occur upon subsequent loading. This opens the door to "mechanical tempering," where the strength of such composites in tension could be increased by inducing residual compressive stresses in the BMG matrix through a uniaxial compressive cycle beyond the onset of plasticity in the reinforcement.

The 5 pct Ta composite (Figure 9(c)) shows a behavior during initial loading similar to the $\mathrm{W}$ composites: the critical von Mises stress at which the first deviation from linearity is observed ( $\sim 200 \mathrm{MPa})$ is around the published value of the uniaxial yield stress of tantalum $\left(165 \mathrm{MPa}^{[15]}\right)$. However, unlike the $\mathrm{W}$ composites, $\sigma_{\text {eff }}$ in the Ta composite again exceeds this uniaxial yield stress upon unloading, so a deflection in the trajectory is expected. This is indeed observed in Figure 9(c) at an applied stress of $600 \mathrm{MPa}$, confirming that the particles have yielded in compression; strain hardening during the first tensile loading may have somewhat increased the yield stress, but the density of data points is too low to ascertain the magnitude of this effect.

As previously mentioned, there was a gap of 7 months between the first loading cycle and the subsequent cycles for the 5 pct Ta composite, and the absolute values of residual strains at the beginning of the second cycle were assumed to be those measured at the end of the first cycle. The particle axial, transverse, and von Mises effective stresses calculated during the second and third loading cycles, however, were exceedingly high compared to those calculated during the first cycle. Based on typical values for BMG viscosities, ${ }^{[1]}$ the BMG matrix should not undergo significant viscous flow under the low residual stresses present after the first cycle, but a possible explanation is partial relaxation of the $\mathrm{Ta}$ particles during the period of time between measurements. The compressive axial and transverse stresses in the particles at the end of the first cycle were -450 and $-160 \mathrm{MPa}$, respectively, as calculated from the residual strains. 


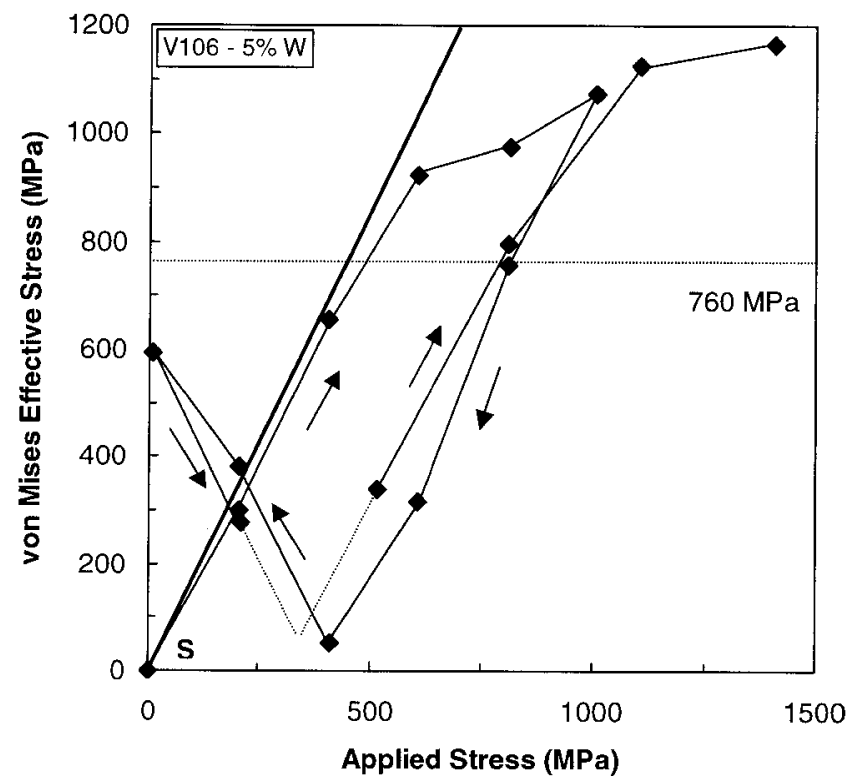

(a)

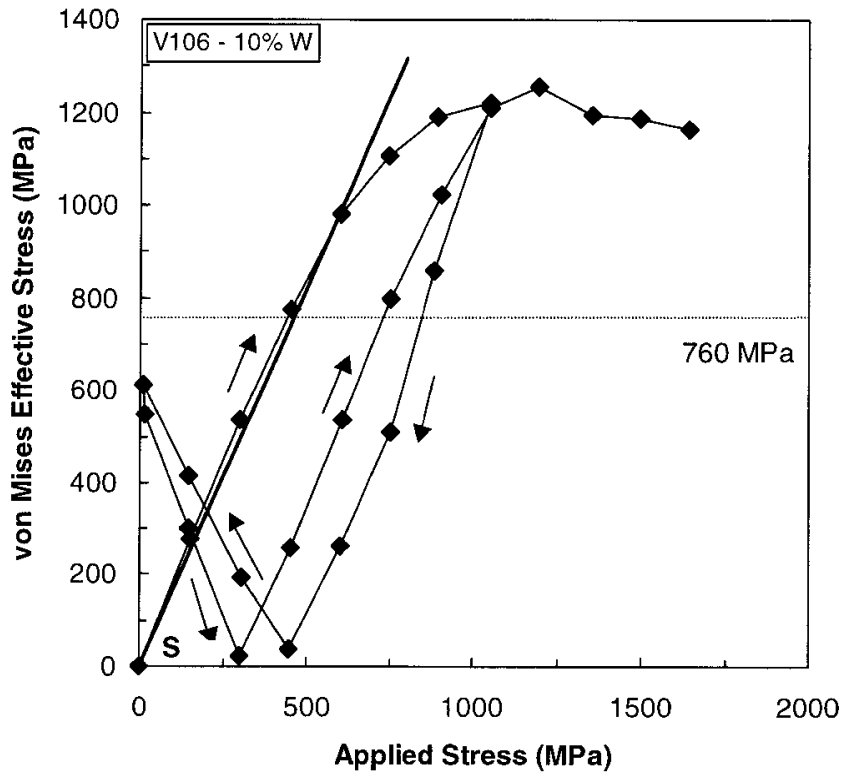

(b)

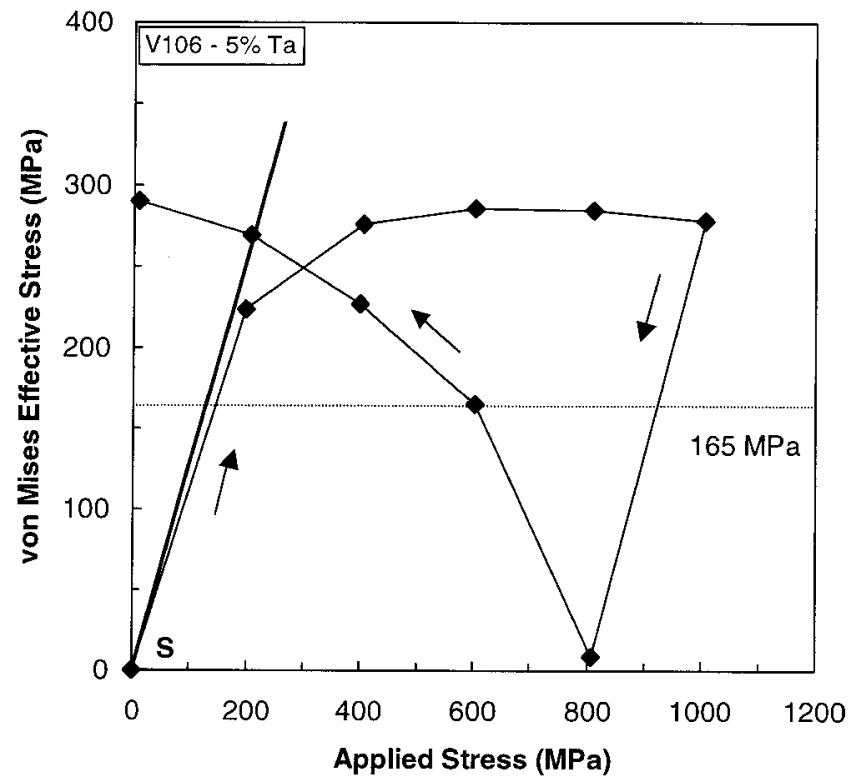

(c)

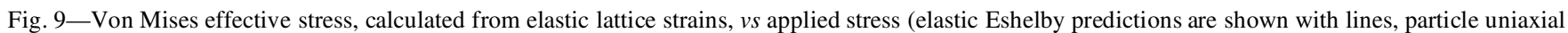
yield stresses from Table I are indicated, and the starting point is marked with an "S"): (a) 5 pct W, (b) 10 pct W, and (c) 5 pct Ta.

The assumption of relaxation of these stresses (e.g., by plasticity or interface slippage) to approximately -250 and $-170 \mathrm{MPa}$ prior to the start of the second cycle, corresponding to shifting the axial and transverse microstrain values by +1100 and $-400 \mu \varepsilon$, respectively, would bring the calculated von Mises stresses back to the levels measured during the first cycle.

\section{Plastic Eshelby Calculations}

To further interpret the plastic behavior of the particles, the Eshelby method was employed, taking into account both elastic and plastic deformation of the reinforcing particles. The nearly spherical shape of the particles, the apparently strong bonding between the two phases, and the low volume fractions of the particles justify the use of this method beyond the elastic regime. Following the scheme proposed in Reference 18, the measured lattice strains in $\mathrm{W}$ and Ta were matched for each applied stress value with the elastic Eshelby prediction by introducing a plastic strain beyond the yield point of the particles. This plastic strain became another component of the transformation strain in the Eshelby calculation in addition to the applied and CTE-mismatch-induced strains. The plastic strain components in the transverse directions were calculated by assuming an effective Poisson's ratio of 0.5 in accordance with the conservation of volume principle, as discussed previously: the value of the axial plastic strain was the only fitting parameter during this calculation. This axial plastic strain is plotted in Figure 10 against the particle effective stress (calculated from measured lattice strains with Eqs. [4] and [5]). 


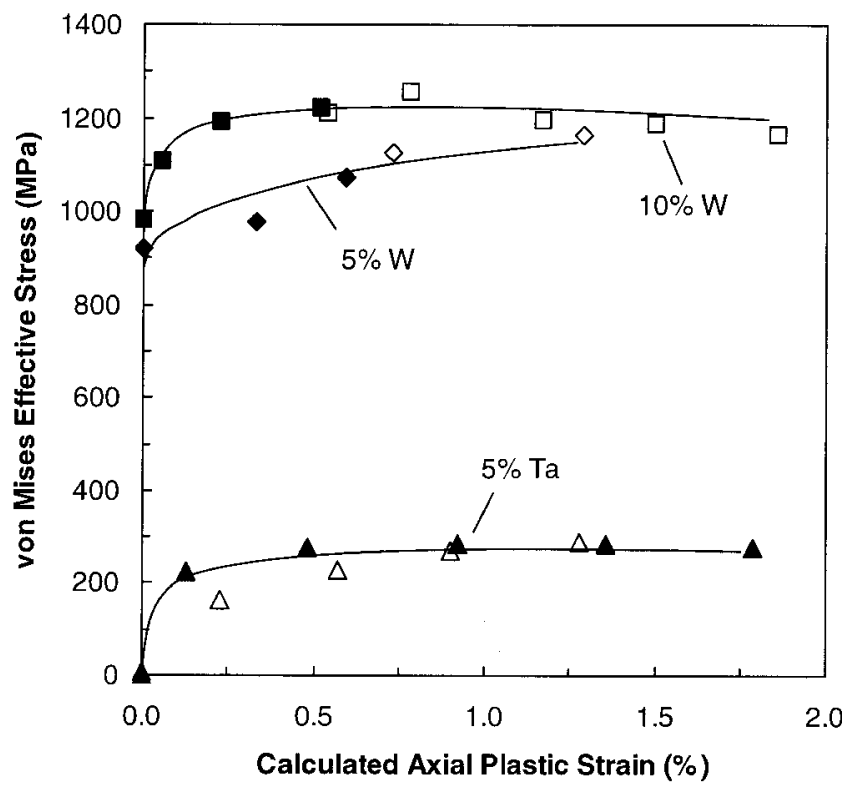

Fig. 10-Von Mises effective stress, calculated from elastic lattice strains, $v s$ particle axial plastic strains, calculated by fitting the measured data to elastic Eshelby predictions (solid symbols correspond to the first loading cycle, and hollow symbols correspond to the second loading cycle (W composites) or the first unloading cycle (Ta composite)). Lines have been added to guide the eye.

The resulting in-situ particle stress-strain curves are qualitatively similar for the 5 and 10 pct W composites, exhibiting rapid strain hardening initially, followed by nearly perfectly plastic behavior at higher plastic strains. The highly constrained plastic deformation of the $\mathrm{W}$ particles is probably responsible for their high ductility. The Ta particles exhibit a qualitatively similar behavior on loading (filled triangles in Figure 10), followed by reverse plasticity and subsequent mild strain hardening during the unloading portion (hollow triangles in Figure 10) of the mechanical cycle. The unloading plastic strains were calculated independently of the loading curve, using the predicted elastic Eshelby unloading strain values as the baseline for calculating the plastic strains and considering the plastic strains as positive irrespective of the tensile or compressive nature of the deformation. The agreement between the loading and unloading Ta curves in Figure 10 is good, indicating that the prior tensile straining during loading had minimal effect on the subsequent compression behavior of the Ta particles. The curves in Figure 10 could be compared to uniaxial stress-strain curves for cast, annealed tungsten or tantalum, which however are not available in the literature. However, the general shape of the stress-strain curves in Figure 10 is reasonable.

In summary, the plastic Eshelby calculations support the earlier observations that the metallic particles yield and strain harden while transferring load to the largely elastic matrix. In the fully elastic regime, the stiffer reinforcement unloads the matrix; after the unavoidable initiation of plasticity in the particles, this benefit is lost and the presence of plastic particles eventually increases, rather than decreases, the stress supported by the elastic matrix. As discussed previously, the average level of "overloading" (difference between average matrix stress and applied stress) at the highest applied stresses is only approximately $20 \mathrm{MPa}$. However, the localized stresses near the matrix-particle interface will be considerably higher and could thus initiate matrix shear banding and subsequent failure. This negative effect must be more than compensated by the beneficial effect of particles inhibiting shear band localization, since the composites reached stresses more than $400 \mathrm{MPa}$ above the unreinforced matrix failure stress.

\section{CONCLUSIONS}

The synchrotron diffraction technique has been used to study the average elastic strain behavior of stiff, elasto-plastic, metallic particles contained within an compliant, elastic, bulk metallic glass matrix during uniaxial tensile loading and unloading of BMG-W and BMG-Ta composites. At low applied stresses, elastic loading of the particles was found to occur in good quantitative agreement with continuum mechanics predictions of load transfer between two well-bonded elastic phases. At higher stresses, plastic deformation of the particles followed by strain hardening was observed, thus reducing the load transfer efficiency from the purely elastic matrix. The strain measurements were used to calculate the von Mises stress in the particles, which was found to deviate from linearity at values near the literature uniaxial yield stress, thus confirming the onset of plasticity. The BMG matrix appears to remain elastic throughout the process. Finally, reinforcement plasticity was found to alter the state of residual strains at zero applied stress. A compressive mechanical cycle is predicted to induce residual compressive stresses in the matrix, a kind of mechanical tempering that may increase the fracture strength of the composites.

\section{ACKNOWLEDGMENTS}

This study was supported by the Center for Structural Amorphous Metals (ARO Grant No. DAAD19-01-0525). DKB gratefully acknowledges the Department of Defense for support in the form of an NDSEG Fellowship. The authors are grateful to Dr. H. Choi-Yim (Caltech) for fabricating all specimens and for helpful discussions, Geoffrey Swift (Caltech) for help with the particle size analysis, and to Dr. H. Choo (Los Alamos National Laboratory) for assistance with the diffraction experiments. All diffraction experiments were performed at the DuPont-Northwestern-Dow Collaborative Access Team (DND-CAT) Synchrotron Research Center located at Sector 5 of the Advanced Photon Source, and the help of the DND-CAT staff is greatly appreciated. DND-CAT is supported by the E.I. DuPont de Nemours \& Co., The Dow Chemical Company, the United States National Science Foundation through Grant No. DMR-9304725, and the State of Illinois through the Department of Commerce and the Board of Higher Education Grant No. IBHE HECA NWU 96. Use of the Advanced Photon Source was supported by the United States Department of Energy, Basic Energy Sciences, Office of Energy Research, under Contract No. W-31-102-Eng-38.

\section{REFERENCES}

1. W.L. Johnson: MRS Bull., 1999, vol. 24, pp. 42-56.

2. R.D. Conner, H. Choi-Yim, and W.L. Johnson: J. Mater. Res., 1999, vol. 14, pp. 3292-97.

3. R.D. Conner, R.B. Dandliker, and W.L. Johnson: Acta Mater., 1999, vol. 46, pp. 6089-6102. 
4. H. Choi-Yim, R.D. Conner, F. Szuecs, and W.L. Johnson: Scripta Mater., 2001, vol. 45, pp. 1039-45.

5. C. Fan, R.T. Ott, and T.C. Hufnagel: Appl. Phys. Lett., 2002, vol. 81, pp. $1020-22$.

6. A.J. Allen, M.A.M. Bourke, S. Dawes, M.T. Hutchings, and P.J. Withers: Acta Metall. Mater., 1992, vol. 40, pp. 2361-73.

7. N. Shi, M.A.M. Bourke, J.A. Roberts, and J.E. Allison: Metall. Mater. Trans. A, 1997, vol. 28A, pp. 2741-53.

8. M.R. Daymond, C. Lund, M.A.M. Bourke, and D.C. Dunand: Metall. Mater. Trans. A, 1999, vol. 30A, pp. 2989-97.

9. A. Wanner and D.C. Dunand: Metall. Mater. Trans. A, 2000, vol. 31A, pp. 2949-62.

10. E. Maire, A. Owen, J.-Y. Buffiere, and P.J. Withers: Acta Mater., 2001, vol. 49, pp. 153-63.

11. M.R. Daymond and P.J. Withers: Scripta Mater., 1996, vol. 35, pp. 1229-34.

12. A. Wanner and D.C. Dunand: J. Neutron Res., 2001, vol. 9, pp. 495-501.
13. H. Choi-Yim: Ph.D. Thesis, California Institute of Technology, Pasadena, CA, 1998

14. Handbook of Elastic Properties of Solids, Liquids, and Gases, Academic Press, San Diego, CA, 2001, vol. 2, p. 100.

15. Metals Handbook, 10th ed, ASM INTERNATIONAL, Materials Park OH, 1990, vol. 2, pp. 576 and 580.

16. I.C. Noyan and J.B. Cohen: Residual Stress-Measurement by Diffraction and Interpretation, Springer-Verlag, New York, NY, 1987, p. 145.

17. D. Dragoi, E. Ustundag, B. Clausen, and M.A.M. Bourke: Scripta Mater., 2001, vol. 45, pp. 245-52.

18. T.W. Clyne and P.J. Withers: An Introduction to Metal Matrix Composites, Cambridge University Press, Cambridge, UK, 1993, pp. 44-70 and 108-12.

19. C.C. Hays, C.P. Kim, and W.L. Johnson: Phys. Rev. Lett., 2000, vol. 84 pp. 2901-04.

20 M.A. Meyers and K.K. Chawla: Mechanical Metallurgy, Prentice-Hall, Inc., Englewood Cliffs, NJ, 1984, pp. 55 and 76. 